\title{
Opposing effects of cationic antimicrobial peptides and divalent cations on bacterial lipopolysaccharides
}

\author{
Matthew Smart, Aruna Rajagopal, ${ }^{*}$ Wing-Ki Liu, ${ }^{\dagger}$ and Bae-Yeun $\mathrm{Ha}^{\ddagger}$ \\ Department of Physics and Astronomy, University of Waterloo, Waterloo, Ontario N2L 3G1, Canada
}

(Received 18 May 2017; revised manuscript received 28 August 2017; published 9 October 2017)

\begin{abstract}
The permeability of the bacterial outer membrane, enclosing Gram-negative bacteria, depends on the interactions of the outer, lipopolysaccharide (LPS) layer, with surrounding ions and molecules. We present a coarse-grained model for describing how cationic amphiphilic molecules (e.g., antimicrobial peptides) interact with and perturb the LPS layer in a biologically relevant medium, containing monovalent and divalent salt ions (e.g., $\mathrm{Mg}^{2+}$ ). In our approach, peptide binding is driven by electrostatic and hydrophobic interactions and is assumed to expand the LPS layer, eventually priming it for disruption. Our results suggest that in parameter ranges of biological relevance (e.g., at micromolar concentrations) the antimicrobial peptide magainin 2 effectively disrupts the LPS layer, even though it has to compete with $\mathrm{Mg}^{2+}$ for the layer. They also show how the integrity of LPS is restored with an increasing concentration of $\mathrm{Mg}^{2+}$. Using the approach, we make a number of predictions relevant for optimizing peptide parameters against Gram-negative bacteria and for understanding bacterial strategies to develop resistance against cationic peptides.
\end{abstract}

DOI: 10.1103/PhysRevE.96.042405

\section{INTRODUCTION}

The bacterial lipopolysaccharide (LPS) is a key surface component of Gram-negative bacteria. It forms the outer layer of the outer membrane $(\mathrm{OM})$, i.e., the first protection layer against potentially harmful molecules such as antimicrobial peptides (AMPs) and antibiotics [1-6] [see Fig. 1(a)]. A distinguishing feature of LPS is its polyanionicity [1-3], each carrying four or more negative charges [Fig. 1(b)]. How LPS molecules interact with other molecules and ions has a profound impact on the LPS layer, which in turn influences OM permeability [Fig. 1(a)]. If $\mathrm{Mg}^{2+}$-binding of LPS creates tightening effects on the LPS layer, EDTA or cationic AMPs reverse these favorable effects by displacing the previously bound $\mathrm{Mg}^{2+}$ ions from the LPS layer, competitively or by chelation, respectively [3-11]. (For more recent progress, see Refs. [12-16].) Indeed, increased OM permeability is correlated with enhanced susceptibility of Gram-negative bacteria to AMPs and antibiotics [9-11].

Because of their OM, Gram-negative bacteria have become increasingly challenging to treat in the clinic (see Ref. [17] and references therein). A better understanding of how AMPs reduce OM permeability will benefit our effort for combating these bacteria. Indeed, several attempts have recently been made to advance our understanding of OM permeability [12-16]. For instance, an in vitro model of the Escherichia coli cell surface has been proposed and used in further unravelling the effects of divalent cations on OM permeability [12-14]. Also, computer modeling has quantified the effects of divalent cations on LPS polymorphism [15].

Despite its potential benefits, theoretical understanding of how the LPS layer is modified by AMPs, in the presence or absence of $\mathrm{Mg}^{2+}$, remains poor. The main bottleneck

\footnotetext{
${ }^{*}$ Current address: Science Institute, University of Iceland, 107 Reykjavik, Iceland.

${ }^{\dagger}$ wkliu@uwaterloo.ca

†byha@uwaterloo.ca
}

in theoretical modeling of LPS is threefold: (i) the longranged nature of Coulomb forces tends to invalidate a simple Langmuir-type binding model [18]; (ii) ionic sizes and peptide area are important molecular details that have to be taken into account [19-23]; (iii) importantly, the hydrophobic binding of (amphiphilic) AMPs into the LPS layer rearranges the surrounding LPS molecules, thus altering the distribution of LPS charges and their electrostatic interactions with peptide charges. Earlier theoretical studies were focused on electrostatic modification of the LPS layer [19,20]. In these studies, the two hurdles (i) and (ii) were overcome; what remains to be explored is LPS perturbation caused by the hydrophobic binding of AMPs. Indeed, the disruption of LPS membranes is correlated with membrane thinning [24]. This suggests that the hydrophobic adsorption of peptides into the membrane, leading to membrane-area expansion, is a prerequisite for membrane disruption [24], as was seen with phospholipid membranes $[25,26]$.

Along this line, it is worth mentioning that a "large-scale" consideration will be beneficial for understanding several essential features of a peptide-membrane system. For instance, it was shown that there exists an optimal charge at which peptide activity is maximized against phospholipid membranes carrying anionic lipids [27,28]. This points to the significance of the mutual electrostatic repulsion between peptides on the membrane surface, which cannot be reduced to single-peptide properties. Also, membrane disruption by peptides is shown to be a "cooperative" process in that it requires a threshold peptide concentration on the membrane surface. This is best understood for phospholipid membranes [25,26], but it also features LPS membranes [24]. If taken altogether, all this means that a physical model of an LPS-peptide-ion system merits much consideration, especially one that will enable us to identify the biophysical or physicochemical parameters for optimal peptide activity against LPS, offering guiding principles for optimizing peptide parameters against Gramnegative bacteria [29].

Here, we present a coarse-grained model for describing competing effects of AMPs and $\mathrm{Mg}^{2+}$ on LPS. To this 
(a)
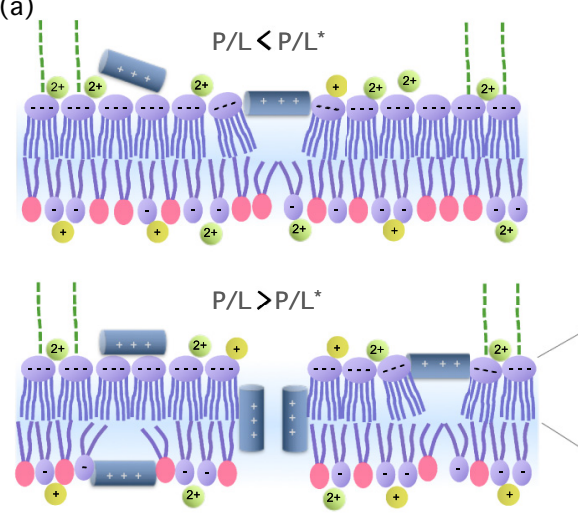

(b)

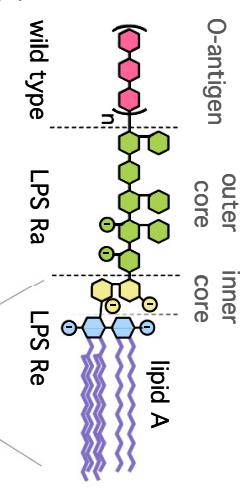

(c)
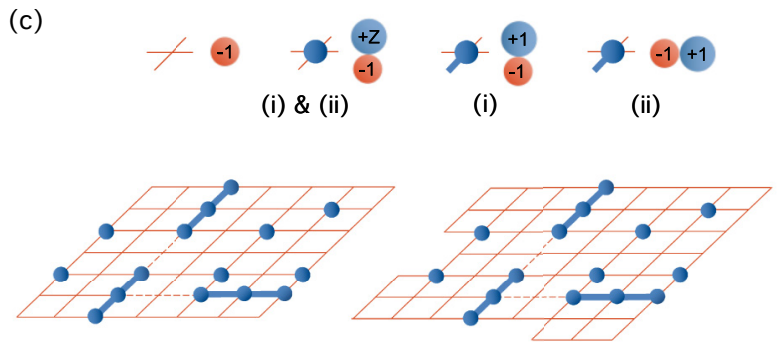

(i) earlier electrostatic binding model: original LPS lattice intact (ii) hydrophobic-electrostatic binding model: LPS-layer expansion and lattice reconstruction

FIG. 1. Outer membrane (OM) permeability in the presence of cationic AMPs and $\mathrm{Mg}^{2+}$, and our modeling strategy. (a) (Upper panel) At a low surface coverage, AMP binding perturbs weakly the outer membrane. Bound peptides reside on the headgroup surface or at the headgroup-tail interface in a parallel orientation. At a sufficiently high coverage, AMPs can disrupt the OM by forming pores, for instance (see Fig. 7 in Ref. [33]). For simplicity, polysaccharides (green dashed lines) are shown for a few LPS molecules. (b) The molecular structure of LPS is shown. Each hexagon represents a sugar molecule: glucosamine in light blue (in lipid A), Kdo in yellow (in the inner core),... The illustration in (b) is adapted from Ref. [34] (see Refs. [1-3] for more details). Copyrighted by the American Physical Society. (c) This shows a surface-lattice model (i), in which LPS charges are viewed as forming discrete lattice sites, decorated with ions and peptides, as well as "lattice reconstruction" (ii). Parallel insertion of a peptide will perturb the lattice. In our approach, this is taken into account by shifting a few lattice sites; the resulting lattice reconstruction is shown [the right bottom panel labeled as (ii)]. Also shown are ion pairs between two opposite charges (e.g., $\mathrm{Mg}^{2+}$ and an LPS charge right below) on the top and area exclusion effects. The dashed line or a short stretch of lattices sites cannot accommodate a peptide in the same orientation. The illustrations in this figure are adapted from Ref. [19] by permission of The Royal Society of Chemistry so as to include pore formation in (a) and hydrophobic insertion, leading to lattice reconstruction in (c).

end, we extend the earlier electrostatic (i.e., surface-lattice) model of LPS [19,20] so as to capture peptide's hydrophobic interactions with LPS and the resulting LPS perturbation. Recall that the hydrophobic adsorption of a peptide into the LPS layer not only rearranges the surrounding LPS molecules but also modifies their electrostatic interaction with the peptide. This poses a major barrier to our theoretical modeling of LPS-AMP interactions. To overcome this, we introduce a "reconstructed-lattice" model, as illustrated in Fig. 1(c).

In our approach, an amphiphilic peptide is modeled as a rectangle; its amphiphilicity is mimicked by assigning a net charge $Q$ (given in units of the elementary charge $e$ ) and a hydrophobic interaction energy $H$. As in earlier studies $[19,20,24,30,31]$, we primarily focus on LPS Re, the simplest form of LPS for survival [1], each carrying four negative charges [see Fig. 1(b)]. Our approach is flexible and can be extended to more realistic LPS, as discussed later (see Sec. IV). In the absence of hydrophobic interactions, the LPS layer is simplified as a two-dimensional electrostatic lattice formed by LPS charges [Fig. 1(c)]. Unlike small cations, which occupy single binding sites, a peptide occupies several sites [19,20]. This gives rise to an unfavorable consequence on peptide binding often referred to as "area exclusion": it is excluded from a short stretch of binding sites, represented by a dashed line in Fig. 1(c) [18,20,32].

Peptide binding, assumed to be in a parallel orientation, inevitably perturbs the surrounding LPS molecules, rearranging their charge distribution. One can invoke the following simplification: a hydrophobically bound peptide shifts $\Omega$ consecutive lattice sites by $a$ (lattice constant), as described in Fig. 1(c); $\Omega a^{2}$ is the area each peptide adds to the LPS layer. This lattice reconstruction allows us to consider both hydrophobic and electrostatic interactions simultaneously.
Using this model, we show how the LPS layer interacts with and is modified by AMPs in an electrolyte solution, possibly containing $\mathrm{Mg}^{2+}$. A central quantity along this line is the molar ratio of hydrophobically bound peptides to lipids often denoted as $P / L$, where $P$ and $L$ can be understood as the number of bound peptides and the number of lipids in a membrane, respectively [24]. In the low micromolar range of this peptide in the bulk, $P / L$ can reach a "threshold" value $P / L^{*}(P / L \approx 0.1$ for magainin 2$)$, beyond which the LPS layer is permeabilized via pore formation or other mechanisms [24,33], as illustrated in Fig. 1(a). Pore formation is not the sole mechanism for disrupting the LPS layer. In our approach, we do not attempt to clarify the mechanism of LPS disruption but focus on extracting general features of our LPS-peptide system. Largely independent of details, LPS perturbation by AMPs can be quantified in terms of $P / L$ or the surface coverage of hydrophobically bound AMPs. A related quantity is the lateral pressure of LPS layers or membranes. Lipid compression by peptide insertion results in an increased lateral pressure, which can be measured experimentally [31].

Our approach shows how AMPs and $\mathrm{Mg}^{2+}$ compete for LPS. It thus explains in physical terms a few key features of long-standing observations regarding how OM permeability can be modified by various OM stabilizing and permeabilizing agents [3-8,24,31]. First, it suggests that at a physiological concentration (typically in a micromolar range) magainin 2 effectively perturbs the LPS layer in an $\mathrm{Mg}^{2+}$-dependent way. For LPS disruption (i.e., $P / L \geqslant P / L^{*}$ ), a larger peptide concentration is required for a larger $\mathrm{Mg}^{2+}$ concentration in the bulk.

Also, it shows how peptide-LPS parameters and peptide activity are interrelated. For instance, it indicates the existence of an optimal peptide charge at which peptide activity is 


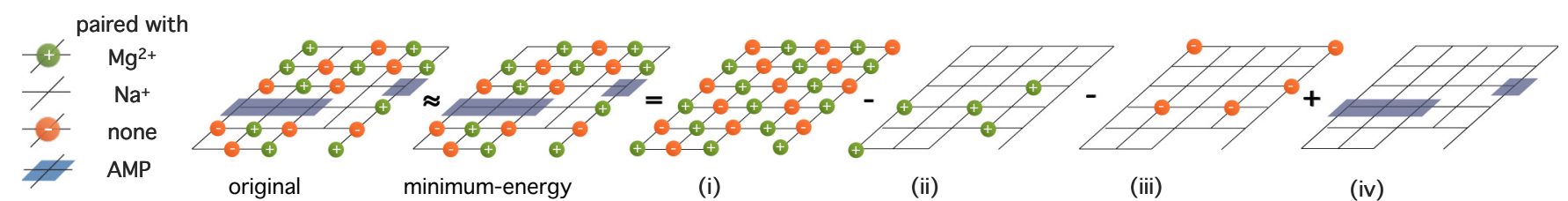

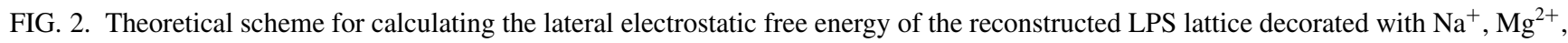
and peptides. Recall that a hydrophobically bound peptide will shift $Q$ lattice sites and create $Q$ sites for its occupation. Except for this, our scheme here is essentially identical to the one used for electrostatically bound peptides $[19,20]$. We first rearrange charges on the lattice into an energy-minimizing distribution in which the charges alternate in sign except on neutral ones $\left(\mathrm{Na}^{+}\right.$-paired or polycation-paired sites). We then use as a reference a "perfect" lattice shown in (i), where equal numbers of positive and negative charges are alternatively arranged. Since this does not correctly represent the initial energy-minimizing one to the left of (i), we remove some of the charges until the perfect lattice becomes the initial one and calculate the resulting free energy cost. In the limit of $N_{0} \rightarrow \infty$, the irregular shape of the reconstructed lattice becomes irrelevant. (This illustration is modified from Refs. [19,20] by permission of The Royal Society of Chemistry; it is consistent with our reconstructed lattice model in Fig. 1(c).)

maximized against LPS. Furthermore, it clarifies to what extent (partial) neutralization of LPS charges weakens AMP's attraction to LPS, lowering $P / L$. This consideration will be beneficial for offering biophysical insights into bacterial strategies for developing resistance against AMPs [10,11,33,35]. Conversely, it establishes the peptide-parameter requirements for enhanced peptide activity against LPS layers with reduced surface charges. The main advantage of our approach is that it can be extended to other cationic peptides, once their biophysical properties are characterized (see Ref. [24] for magainin 2-LPS interactions). Its variation will be useful for clarifying the peptide parameters for optimal peptide activity against Gram-negative bacteria.

\section{THEORETICAL APPROACH}

We first derive the free energy of our LPS system. To this end, we extend the electrostatic model of LPS [19,20], in which molecular binding is purely electrostatically driven, to one that captures the hydrophobic binding of peptides to LPS and the resulting LPS perturbation. In our approach, we assume that all bound peptides are hydrophobically associated with LPS. This is reasonable, since an inserted one gains a large hydrophobic free energy $\left(\approx-10 k_{\mathrm{B}} T\right)$, where $k_{\mathrm{B}}$ is the Boltzmann constant and $T$ the temperature. The resulting lattice perturbation is taken into account via the lattice reconstruction mentioned earlier: a bound peptide shifts $\Omega$ consecutive lattice sites and creates $\Omega$ sites for its own occupancy [Fig. 1(c)] [36]. In this picture, the lattice constant will remain unchanged, as long as the bilayer coupling is ignored; also, $\Omega a^{2}$ should be interpreted as the area each peptide adds to the LPS layer. The main difference is that the sites previously representing LPS charges have been replaced by peptide charges.

Our approach does not take into account explicitly the eventual disruption of LPS layers beyond $P / L^{*}$, for example, via pore formation. Instead, we will show how $P / L^{*}$ is reached as the peptide concentration increases.

The electrostatic free energy of our LPS system can be expressed in terms of several parameters. Let $N_{0}$ be the number of original lattice sites, which is four times the number of LPS molecules (recall each LPS Re carries four negative charges), $N_{1}, N_{2}$, and $N_{p}$ the number of $\mathrm{Na}^{+}$ions, $\mathrm{Mg}^{2+}$ ions, and AMPs, on the lattice, respectively. (Note that $N_{p}$ and $P$ as in $P / L$ can be used interchangeably. Here we introduced a few similar parameters: $N_{1}$ and $N_{2}$. Except for the ratio $P / L$, we preferentially use $N_{p}$ over $P$.) These are the parameters with respect to which the total free energy is to be minimized. The number of reconstructed lattice sites is then $\tilde{N}_{0} \equiv N_{0}+\Omega N_{p}$.

The total electrostatic energy of a given charge arrangement (e.g., the "original" one in the left corner in Fig. 2) is given as a pairwise sum of two-body interactions. This allows one to decompose the LPS free energy into a few components: ion pairing energy, lateral interactions (or simply lateral correlations), and a net charge repulsion [19,20]. Among them, the lateral interaction is the most formidable to derive, since it should reflect nonuniform charge distributions on the LPS layer (see the Appendix of Ref. [19] for the details).

Here, we focus on describing how the earlier electrostatic lattice model can be extended to our reconstructed-lattice model [Fig. 1(c)]. Let $N_{ \pm}$be the number of positive (green balls) or negative charges (tangerine balls) on the original lattice in the left corner in Fig. 2. Obviously, $N_{+}=N_{2}$ and $N_{-}=N_{0}-N_{1}-N_{2}-Q N_{p}$. First, let us ignore ion pairing between opposite charges and focus on the lateral interactions. As illustrated in Fig. 2, we first approximate the original charge distribution into an energy-minimizing one in which the charges alternate in sign except on neutral ones $\left(\mathrm{Na}^{+}\right.$-paired or polycation-paired sites). It proves useful to construct a "perfect," alternating lattice, labeled as (i) in Fig. 2, and expand the lateral free energy $F_{\text {lat }}$ about this arrangement. Since this does not correctly represent the initial one we start with, i.e., the one to the left of (i), we remove some of the charges until the perfect lattice becomes the initial (energy-minimizing) distribution and calculate the resulting free energy cost.

Let $\Sigma_{\text {alt }}$ be the electrostatic interaction of a given charge on the perfect, alternating lattice (i), which is the same for negative and positive charges on the lattice. The lateral free energy per site $F_{\text {lat }}$ can be written as $[19,20]$

$$
F_{\text {lat }} \tilde{N}_{0} \approx \frac{\tilde{N}_{0}}{2} \Sigma_{\text {alt }}-M \Sigma_{\text {alt }}+F_{r r},
$$

where $M=\tilde{N}_{0}-N_{+}-N_{-}$is the number of removed charges and $F_{r r}$ is the interaction free energy among all the removed (or added) charges, i.e., those in (ii)-(iv) in Fig. 2. Here and below, free energy is given in units of the thermal energy $k_{\mathrm{B}} T$.

One can find $\Sigma_{\text {alt }}=-\ell_{\mathrm{B}} \times \Delta_{\varepsilon} \times \zeta(a)$ [19]. Recall that $a$ is the lattice constant. Here, the Bjerrum length $\ell_{\mathrm{B}}$ is given by $\ell_{\mathrm{B}}=e^{2} / 4 \pi \varepsilon_{0} \varepsilon_{r} k_{\mathrm{B}} T$, where $e$ is the electronic 
charge, $\varepsilon_{0}$ the permittivity of free space, and $\varepsilon_{r}$ the dielectric constant of a solvent $\left(\varepsilon_{r}=\varepsilon_{w}\right.$ for water) (see for instance Ref. [18]). The symbol $\Delta_{\varepsilon}$ accounts for the effect of a dielectric discontinuity between water and LPS. Generally, electrostatic interactions near an interface between two dielectric media have a complicated dependence on the dielectric constants of the media (see for instance Ref. [37] and references therein). However, note that $\varepsilon_{w} / \varepsilon_{l} \gg 1$, where $\varepsilon_{l} \approx 2$ is the dielectric constant of lipids. This effect can be factored out as in Eq. (1); for practical purposes, it can be approximated as $\Delta_{\varepsilon} \approx 2$ [38].

Finally, $\zeta(a)$ is an infinite sum given by

$$
\zeta(a)=\frac{1}{2} \sum_{i=1}^{\infty} \sum_{j=0}^{i}(-1)^{i+j-1} \frac{e^{-\kappa a \sqrt{i^{2}+j^{2}}}}{a \sqrt{i^{2}+j^{2}}} \times k(i, j),
$$

where $k(i, j)$ is defined as $k(i, j)=4$, if $j=0$ or $i=j$; $k(i, j)=8$, otherwise [19]. The symbol $\kappa$ is the reciprocal of the screening length, beyond which the electrostatic interactions are exponentially screened. Assume that there are (1:1) and (2:1) salts in the solution, and let $n_{1}$ and $n_{2}$ be the bulk concentration of monovalent and divalent cations, respectively. It is given by $\kappa^{2}=4 \pi \ell_{B}\left[2 n_{1}+\left(2^{2}+2\right) n_{2}\right]$.

Following Refs. $[19,20]$ and using the illustration in Fig. 2, we arrive at

$$
\begin{aligned}
F_{r r} \approx & \frac{2}{\tilde{N}_{0}}\left(\frac{\tilde{N}_{0}}{2}-N_{+}\right)\left(\frac{\tilde{N}_{0}}{2}-N_{-}\right) \Sigma_{\text {alt }} \\
& +\frac{\pi}{\kappa} \frac{\ell_{\mathrm{B}}}{a^{2}} \Delta_{\varepsilon} \frac{\left(N_{+}-N_{-}\right)^{2}}{\tilde{N}_{0}} .
\end{aligned}
$$

The second term on the right-hand side in Eq. (3) represents the net charge repulsion on the LPS layer. Because of peptide binding, this term is reduced by the factor $N_{0} / \tilde{N}_{0}$. If used in Eq. (1), this leads to

$$
\begin{aligned}
F_{\text {lat }} \tilde{N}_{0} \approx & \ell_{\mathrm{B}} \Delta_{\varepsilon}\left[\frac{\pi}{\kappa} \frac{\left(N_{0}-N_{1}-2 N_{2}-Q N_{p}\right)^{2}}{\tilde{N}_{0} a^{2}}\right. \\
& \left.-2 \frac{N_{2}\left(\tilde{N}_{0}-N_{1}-N_{2}-Q N_{p}\right)}{\tilde{N}_{0}} \zeta(a)\right] .
\end{aligned}
$$

Equation (4) can be improved by correcting it for ion pairing between opposite charges: an LPS charge and its ion pair [see the top illustration in Fig. 1(c)]. Let $\delta_{i}(i=1,2)$ and $\delta_{p}$ be the distance between the two opposite charges forming an ion pair, where the subscripts " 1 ," " 2 ," and " $p$ " refer to an LPS charge paired with $\mathrm{Na}^{+}, \mathrm{Mg}^{2+}$, and a peptide charge, respectively. The total ion-pairing free energy can be expressed as $[19,20,22]$

$$
F_{\text {ion pair }}=\ell_{\mathrm{B}} \Delta_{\varepsilon}\left(\frac{N_{1}}{\delta_{1}}+\frac{2 N_{2}}{\delta_{2}}+\frac{Q N_{p}}{\delta_{p}}\right) .
$$

Since the first term on the right-hand side of Eq. (4) includes the continuum analog of this, one needs to be careful to avoid double-counting of this effect [22] [see below Eq. (8)].

As illustrated in Fig. 1(c), a short stretch of free sites $(<$ $\Omega$ ), described by a dashed line, cannot accommodate a chain molecule of length $\Omega$ (in units of $a$ ) in the same orientation. This area-exclusion effect, arising between peptides, can be included within the framework of scaled particle theory [32] (see also Ref. [20] for additional details). An earlier study shows that this effect can be significant in the parameter range of biological relevance [20].
Perhaps, the easiest way to take into account this effect is to start from the case with peptides only. As discussed in Ref. [20], the entropic chemical potential of this system can be obtained from Ref. [32]. In units of $k_{\mathrm{B}} T$, it reads

$$
\begin{gathered}
\mu_{p}=\ln \frac{\Omega N_{p} / \tilde{N}_{0}}{1-\Omega N_{p} / \tilde{N}_{0}}+\frac{1}{1-\Omega N_{p} / \tilde{N}_{0}} \\
+\frac{\epsilon}{\left(1-\Omega N_{p} / \tilde{N}_{0}\right)^{2}}-1-\epsilon .
\end{gathered}
$$

Here $\epsilon$ is the "shape parameter" [32]. For a rectangle of aspect ratio $g, \epsilon=(g / \pi) \times(1+1 / g)^{2} ; \epsilon=\frac{25}{4 \pi}$ for $g=4$, as for magainin 2 , typically carrying four positive charges [32]. Also note that $\tilde{N}_{0}$ is used in place of $N_{0}$ in the original derivation [20]. The first term on the right-hand side would be obtained if one assumed a Langmuir-type model in which area exclusion is ignored [18]. The rest of the terms cancel each other in the limit $N_{p} / N_{0} \rightarrow 0$ but increase rapidly with $N_{p} / N_{0}$; they account for the effect of area exclusion. Since ions do not give rise to area exclusion effects, one can combine their contributions with the first term in Eq. (6) [20].

The hydrophobic free energy of bound peptides can readily be included, if we note that it is set by single-peptide properties. If $H(<0)$ is the hydrophobic free energy per peptide, the total hydrophobic free energy of bound peptides can be expressed as $N_{p} H$.

Finally, we include the free energy cost for LPS deformations induced by peptide binding. Unfortunately, how LPS layers respond to inclusions (e.g., hydrophobically bound peptides) has not been well understood (see Ref. [39] for a similar issue for phospholipids). Nevertheless, one can map out a plausible picture of LPS deformations based on the two observations: (i) the compression modulus of LPS layers is comparable to that of phospholipid layers [16,40,41] (see Sec. III for more details); (ii) both LPS and phospholipid bilayers require a threshold peptide coverage (i.e., $P / L^{*}$ ) for pore formation [24-26]. The latter (ii) is a clear indication that the deformation free energy is quadratic with $N_{p}$ for the biologically relevant range of $P / L\left(=Q N_{p} / N_{0}\right.$ for $Q=4): P / L \lesssim P / L^{*}$ or $P / L \gtrsim P / L^{*}$. This is a natural consequence of LPS deformations that overlap (see Ref. [39] for phospholipids). Otherwise, bound peptides would either remain on the surface in a parallel orientation or prefer to form pores for all values of $P / L$. This contradicts observations [24] (see Refs. [25,26] for phospholipids). Similarly to what was seen with phospholipid membranes, the disruption of LPS occurs beyond $P / L^{*}$, but not below $P / L^{*}$. In other words, around $P / L^{*}$, the deformation free energy should be quadratic with $N_{p}$.

Let $K_{\mathrm{A}}$ be the area compression modulus of LPS layers, which can be measured experimentally. The quadratic deformation free energy (in units of $k_{\mathrm{B}} T$ ) can be expressed as

$$
F_{\text {deform }}\left(N_{p}\right)=\frac{a^{2}}{2} K_{\mathrm{A}} \frac{\left(\Omega N_{p}\right)^{2}}{N_{0}} .
$$

For small $N_{p}$, however, the deformation free energy is expected to be linear with $N_{p}$, since the effect of peptide binding is local in this case. To test the applicability of the quadratic deformation free energy in Eq. (7), in the Appendix, we use an analogy between LPS and phospholipid membranes. 
To this end, we examine a "hypothetical LPS layer," for which the deformation free energy is identical to the one derived for phospholipids that shows a crossover between the linear and quadratic limits [39]. Our analysis in the Appendix suggests that for practical purposes the quadratic
LPS deformation free energy can be used for a wide range of $N_{p} / N_{0}$.

The procedures described above lead to the following total LPS free energy $F_{\text {LPS }}^{\text {total }}$ as a function of $N_{1}, N_{2}$, and $N_{p}$, with reference to the bulk:

$$
\begin{aligned}
F_{\mathrm{LPS}}^{\mathrm{total}}\left(N_{1}, N_{2}, N_{p}\right)= & \ell_{\mathrm{B}} \Delta_{\varepsilon} \cdot\left[\left(\frac{\pi}{\kappa}-\frac{\mathcal{M}_{1}}{2}\right) \frac{\left(N_{0}-N_{1}-2 N_{2}-Q N_{p}\right)^{2}}{\tilde{N}_{0} a^{2}}-\frac{\left(\mathcal{M}_{p}-\mathcal{M}_{1}\right)}{2} \frac{Q N_{p}\left(N_{1}+2 N_{2}+Q N_{p}\right)}{\tilde{N}_{0} a^{2}}\right. \\
& \left.-\frac{N_{1}}{\delta_{1}}-\frac{2 N_{2}}{\delta_{2}}-\frac{Q N_{p}}{\delta_{p}}-2 \frac{N_{2}\left(\tilde{N}_{0}-N_{1}-N_{2}-Q N_{p}\right)}{\tilde{N}_{0}} \zeta(a)\right]+N_{p} H+\frac{a^{2}}{2} K_{\mathrm{A}} \frac{\left(\Omega N_{p}\right)^{2}}{N_{0}} \\
& +\sum_{i=1}^{2} N_{i} \ln \left(\frac{N_{i}}{\tilde{N}_{0} n_{i} v_{i}}\right)+N_{p} \ln \left(\frac{\Omega N_{p}}{\tilde{N}_{0} n_{p} v_{p}}\right)+\left(\tilde{N}_{0}-N_{1}-N_{2}-\Omega N_{p}\right) \ln \left(1-\frac{N_{1}+N_{2}+\Omega N_{p}}{\tilde{N}_{0}}\right) \\
& +\frac{1-\Omega}{\Omega}\left(\tilde{N}_{0}-\Omega N_{p}\right) \ln \left(1-\frac{\Omega N_{p}}{\tilde{N}_{0}}\right)-N_{p}(\epsilon+1)-\frac{\tilde{N}_{0}}{\Omega} \ln \left(1-\frac{\Omega N_{p}}{\tilde{N}_{0}}\right)+\frac{\tilde{N}_{0}}{\Omega} \frac{\epsilon}{1-\Omega N_{p} / \tilde{N}_{0}} \\
& -\sum_{i} N_{i} \mu_{i}^{\text {free }}-N_{p} \mu_{p}^{\text {free }} .
\end{aligned}
$$

Here, $\mathcal{M}_{v}(v=1$ or $Q)$ is defined as

$$
\mathcal{M}_{\nu}(\kappa, a)=\int_{-a v / 2}^{a v / 2} d x \int_{-a / 2}^{a / 2} d y \frac{e^{-\kappa \sqrt{x^{2}+y^{2}}}}{\sqrt{x^{2}+y^{2}}} .
$$

The first line in Eq. (8) describes the mean-field free energy. The terms containing $\mathcal{M}_{v}$ are to correct the electrostatic free energy for over-counting Coulomb interactions (within the same site), as noted earlier [19,20,22]. All the terms in the second line containing $\delta_{i}$ are the ion-pairing (or transverse) interactions [22,23]. The next one containing $\zeta(a)$ is the lateral interaction. The next two terms represent hydrophobic interactions and membrane deformations induced by peptide binding (recall $H$ is the hydrophobic free energy per peptide and $K_{\mathrm{A}}$ the area-stretch modulus of the LPS layer).

The entropic free energy including the effect of area exclusion is presented in the following two lines. It is expressed with respect to ions and peptides in the bulk. In this, $n_{i}$ and $v_{i}$ are the bulk concentration and the volume of ions, respectively; $n_{p}$ and $v_{p}$ are similarly defined for peptides.

Finally, the energy contributions to the chemical potentials of free ions and peptides (in units of $k_{\mathrm{B}} T$ ) can be obtained as

$$
\begin{aligned}
\mu_{i}^{\text {free }} & =-\frac{Z_{i}^{2}}{2} \frac{\ell_{\mathrm{B}}}{\delta_{i}}\left(\Delta_{\varepsilon}-1\right)-\frac{Z_{i}^{2}}{2} \frac{\ell_{\mathrm{B}} \kappa}{1+\kappa r_{i}}, \\
\mu_{p}^{\text {free }}= & -\frac{Q}{2} \ell_{\mathrm{B}}\left(\Delta_{\varepsilon}-1\right)\left(\frac{1}{\delta_{p}}+\frac{\mathcal{M}_{p}-\mathcal{M}_{1}}{a^{2}}\right) \\
& -\frac{Q}{2} \frac{\ell_{\mathrm{B}} \kappa}{1+\kappa r_{1}} .
\end{aligned}
$$

In Eq. (10), $Z_{1}=1$ and $Z_{2}=2$ refer to monovalent and divalent cations, respectively. Note that the entropic contribution is absorbed into Eq. (8). The first term on the right-hand side of Eq. (10) or (11) is the "self-energy" of free charges with reference to the LPS layer; the first term in Eq. (11) also contains a contribution from the interaction between different charges on the peptide described by the term containing
$\left(\mathcal{M}_{p}-\mathcal{M}_{1}\right)$ [19]. This term is constant if $\varepsilon_{r}$ is constant but varies near a dielectric discontinuity and can be interpreted as an image-charge effect. The second term is the "polarization" energy, i.e., the electrostatic free energy gain of a charged particle due to the shielding of its charge by the surrounding ions $[18,42]$.

So far, we have ignored the bilayer coupling, i.e., the constraint that the outer LPS and inner layers form a bilayer. For the case depicted in Fig. 1(a) (upper panel), the inner layer will resist stretching of the outer one. This can be taken into account, if we assume that both layers have the same or similar elastic properties. Then the stress caused by peptide insertion will be equally distributed between the two layers. Let $a_{0}$ be the "unperturbed" lattice constant in the absence of peptide binding in a high-salt limit. If we assume an asymmetric binding of AMPs as in Fig. 1(a) (upper panel), the lattice constant is modified as [43]

$$
a\left(N_{p}\right)=\frac{a_{0}}{\sqrt{1+c \Omega N_{p} / N_{0}}} .
$$

Here $c=1 / 2$ for an asymmetric binding. For an LPS monolayer with the total area held fixed, $c=1$. For a symmetric binding between the two layers as in an LPS bilayer, however, $a$ can be approximated as $a_{0}$. This will simplify the analysis of the LPS free energy in Eq. (8). In this work, for simplicity, we focus our effort on analyzing a symmetric LPS bilayer, in which AMPs are symmetrically distributed between the inner and outer layers; it suffices to focus on one of the layers, e.g., the outer layer. The difference between symmetric and asymmetric cases is only subtle and quantitative. The symmetric case is, however, much easier to analyze numerically.

The lateral pressure change due to insertion is given by [44]

$$
\Delta \Pi=-\frac{\partial F_{\mathrm{LPS}}^{\mathrm{total}}}{\partial a^{2}}+K_{\mathrm{A}} \frac{\Omega N_{p}}{N_{0}} \approx K_{\mathrm{A}} \frac{\Omega N_{p}}{N_{0}} .
$$




\section{Fractional Area Expansion (A) and Lateral Pressure (B)}
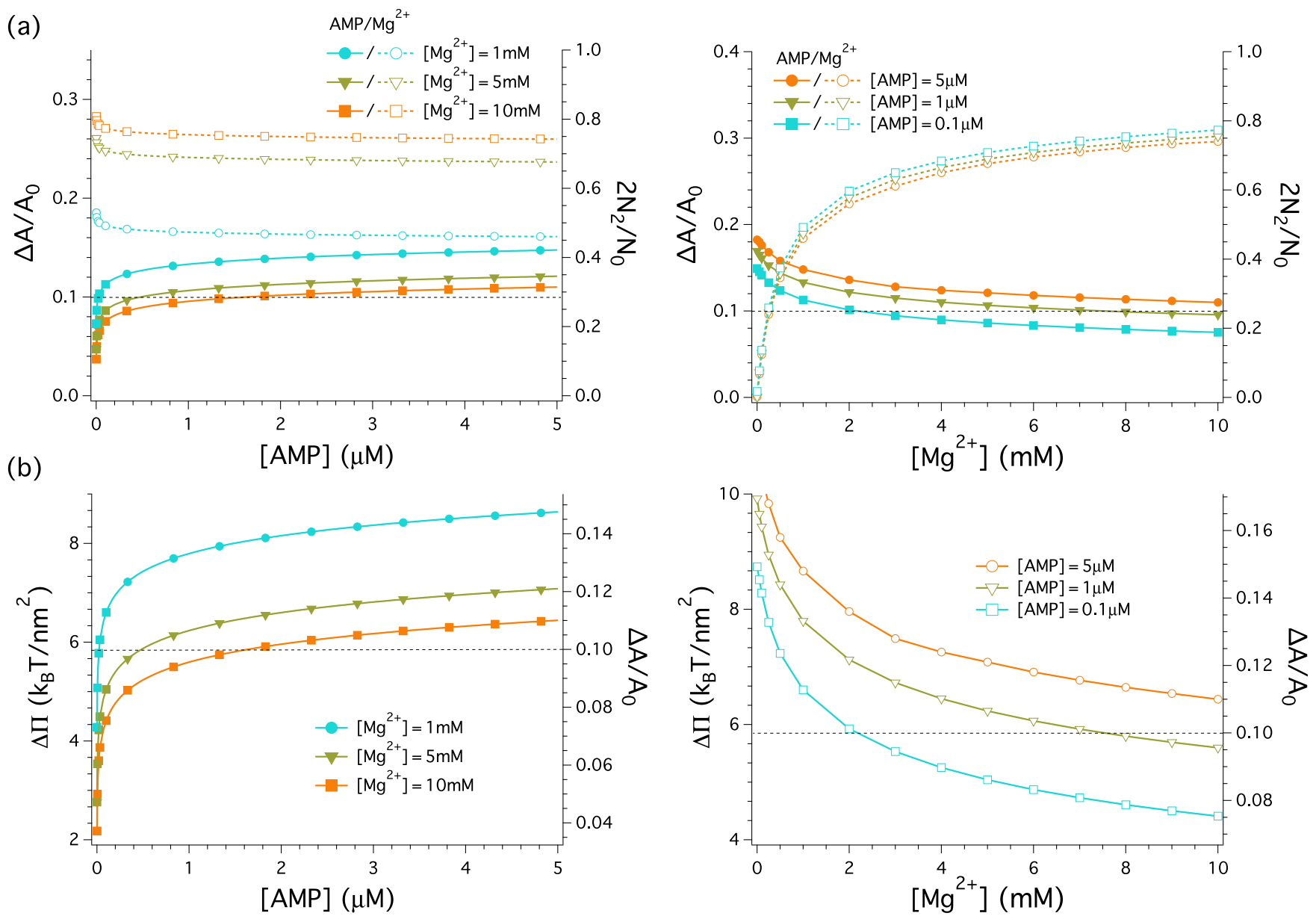

FIG. 3. Competitive binding of $\mathrm{Mg}^{2+}$ and AMPs to LPS as well as LPS perturbation. We have chosen $\left[\mathrm{Na}^{+}\right]=100 \mathrm{mM}$ and $Q=4$, and used a few choices of $\left[\mathrm{Mg}^{2+}\right]$ (left) and [AMP] (right) as indicated in the legend. (a) The graph shows the fractional area increase of LPS, $\triangle A / A_{0}$, and the fractional charge occupancy of $\mathrm{Mg}^{2+}$ on the LPS layer, $2 N_{2} / N_{0}$; for $\Omega=Q=4$ as for magainin $2, \Delta A / A_{0}=Q N_{p} / N_{0}=P / L$, i.e., the fractional area increase coincides with the fractional charge occupancy of AMPs. (Left) As [AMP], the bulk concentration of AMPs, increases, the occupancy of AMPs on the LPS layer and $\triangle A / A_{0}$ increase, reducing the binding of $\mathrm{Mg}^{2+}$. This graph suggests that LPS perturbation by AMPs is more effective for a smaller value of $\left[\mathrm{Mg}^{2+}\right]$. (Right) As $\left[\mathrm{Mg}^{2+}\right]$, the bulk concentration of $\mathrm{Mg}^{2+}$, increases, the occupancy of $\mathrm{Mg}^{2+}$ on the LPS layer increases, diminishing the binding of AMPs. This graph implies that the LPS stabilizing effects of $\mathrm{Mg}^{2+}$ is more pronounced for a smaller value of [AMP]. (b) These graphs show the excess lateral pressure $\triangle \Pi$ of the LPS layer induced by peptide binding. Note that $1 k_{\mathrm{B}} T / \mathrm{nm}^{2} \approx 4.1 \mathrm{mN} / \mathrm{m}$ at $300 \mathrm{~K}$. (Left) The increase of $\Delta \Pi$ with [AMP] is correlated with the [AMP] dependence of $\Delta A / A_{0}$ in (a). As [AMP] increases, more peptides will bind to LPS, increasing $\Delta \Pi$. (Right) This shows how $\Delta \Pi$ varies with $\left[\mathrm{Mg}^{2+}\right]$. Obviously, it decreases as $\left[\mathrm{Mg}^{2+}\right]$ increases. This is correlated with the enhanced binding of $\mathrm{Mg}^{2+}$ at higher concentrations seen in (a). The trend seen with the curves corresponding to different values of $\left[\mathrm{Mg}^{2+}\right]$ on the left is consistent with the $\mathrm{Mg}^{2+}$-dependence of the results in the graphs on the right.

Here, $F_{\mathrm{LPS}}^{\text {total }}$ is the LPS free energy already minimized with respect to $N_{i}$ and $N_{p}$; the partial derivative with respect to $a$ is to be evaluated at $a=a_{0}$. The main advantage of absorbing the entropy of free ions or peptides into $F_{\mathrm{LPS}}^{\text {total }}$ in Eq. (8) is that the resulting $F_{\mathrm{LPS}}^{\text {total }}$ correctly captures the entropic gain of ions or peptides in the computation of $\partial F_{\mathrm{LPS}}^{\text {total }} / \partial a^{2}$. The next term represents the free energy cost for membrane deformation. The second equality holds for a parameter space of biological interest: $\Omega N_{p} / N_{0} \approx Q N_{p} / N_{0} \approx 0.1$.

In equilibrium, $F_{\mathrm{LPS}}^{\text {total }}$ is minimized with respect to $N_{i}$ and $N_{p}$, or $\mu_{i}^{\text {free }}=\mu_{i}^{\mathrm{LPS}}=\partial F_{\mathrm{LPS}}^{\text {total }} / \partial N_{i}$ and $\mu_{p}^{\text {free }}=\mu_{p}^{\mathrm{LPS}}=$ $\partial F_{\mathrm{LPS}}^{\text {total }} / \partial N_{p}$. These relations can be solved simultaneously for $N_{i}$ and $N_{p}$.

\section{RESULTS}

In our analysis, we have chosen the parameters as follows: $\left[\mathrm{Na}^{+}\right]=100 \mathrm{mM}\left(\kappa^{-1} \approx 10 \AA\right), \delta_{1}=\delta_{p}=3 \AA, \delta_{2}=2.5 \AA$, $r_{1}=3.4 \AA, r_{2}=4.3 \AA$, and $T=300 \mathrm{~K}[45,46]$. As for peptide parameters, we have used typical values for magainin 2 [10,24], unless otherwise indicated: $Q=4$ (Fig. 3) and $v_{p}=$ $2.5 \mathrm{~nm}^{3}$ [47]; a reasonable choice of $\Omega$ is $\Omega \approx Q=4$, as for magainin 2 [48]. To determine $a_{0}$, the unperturbed lattice constant (i.e., when the electrostatic interaction is "turned off" or screened sufficiently), note that the typical cross-sectional area of LPS Re $\approx 166 \AA^{2}[30]$. We thus use $a_{0}=\sqrt{166 / 4}=$ $6.44 \AA$. (In Fig. 4, however, we vary $Q$ and $a_{0}$ to examine their effects on LPS.) Finally, we have chosen $H=-10 k_{\mathrm{B}} T$ and 


\section{Fractional Area Expansion vs. $\mathrm{Q}(\mathrm{A} \& \mathrm{C})$ and $\mathrm{a} / \mathrm{a}_{0}(\mathrm{~B})$}

(a)

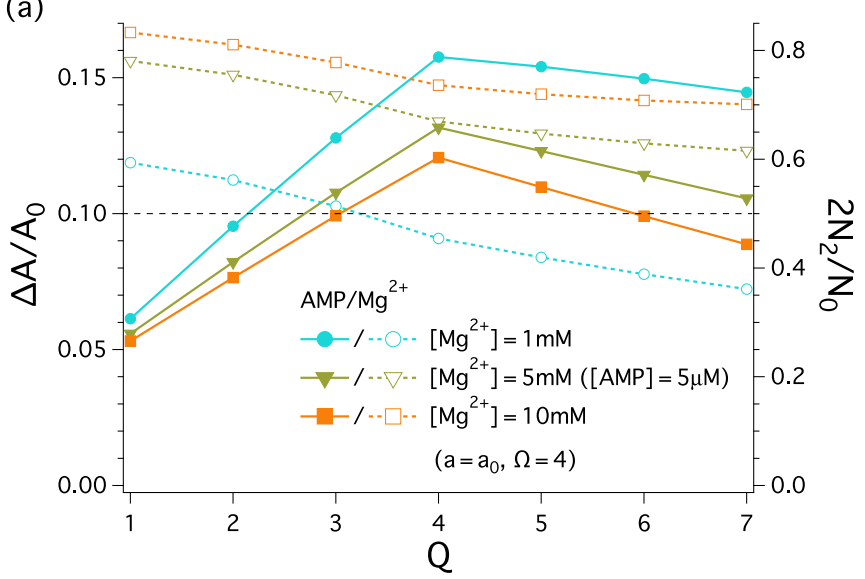

(b)

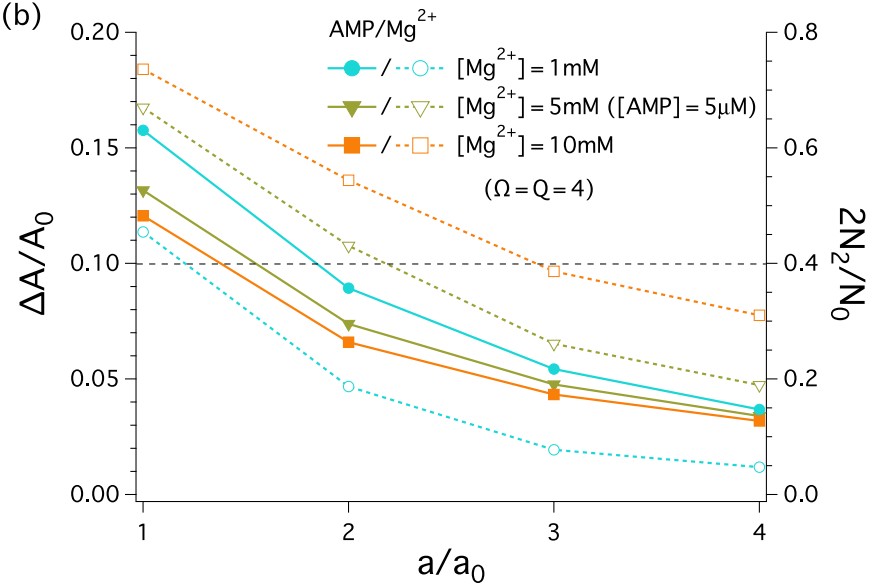

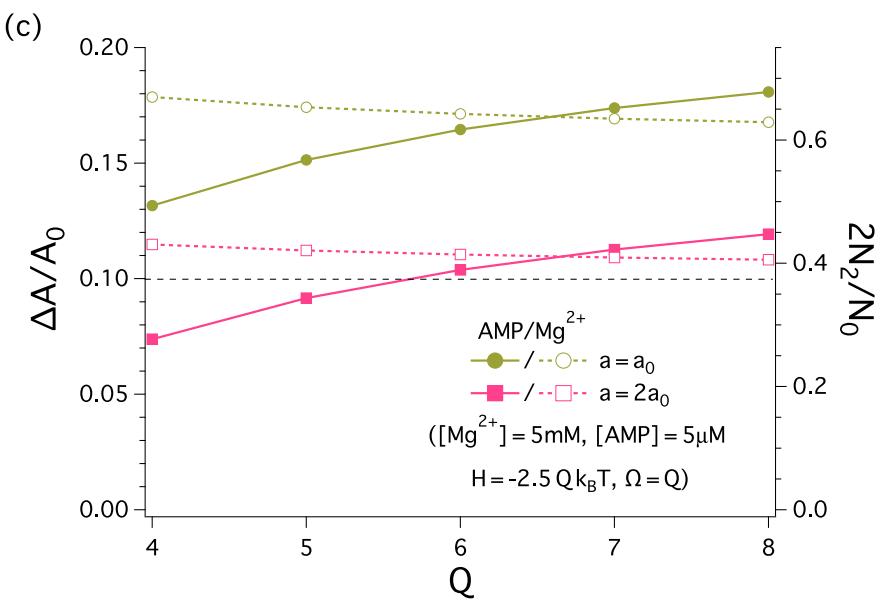

FIG. 4. Fractional area change, $\Delta A / A_{0}$, and fractional charge occupancy of $\mathrm{Mg}^{2+}, 2 N_{2} / N_{0}$ vs. $Q$ (a and c) and $a / a_{0}$ (b). We have chosen $H=-10 k_{\mathrm{B}} T$ and $\Omega=4$ in both (a) and (b); $Q=1, \ldots, 7$ in (a) and $Q=4$ in (b); in (b), the lattice constant is chosen to be $a=1,2,3,4 a_{0} ;$ in (c), $Q=\Omega$ and $H=-2.5 Q k_{\mathrm{B}} T\left(=10 k_{\mathrm{B}} T\right.$ for $\Omega=4$ ); in all cases, $\left[\mathrm{Na}^{+}\right]=100 \mathrm{mM}$. (a) The graph shows the results for $\Delta A / A_{0}=\Omega N_{p} / N_{0}$ and $2 N_{2} / N_{0}$. (a) As $Q$ increases, $\Delta A / A_{0}$ increases initially and then decreases beyond $Q=4$, for all [ $\left.\mathrm{Mg}^{2+}\right]$ values used. On the other hand, $2 N_{2} / N_{0}$ decreases monotonically. The non-monotonic dependence of $\Omega N_{p} / N_{0}$ on $Q$ can be attributed to the balance between LPS-peptide attractions and peptide-peptide repulsions on the LPS surface, both becoming stronger with increasing $Q$. (b) As the lattice dilates, both $\Delta A / A_{0}$ and $2 N_{2} / N_{0}$ decrease monotonically, as their attraction with LPS diminishes. In all the cases shown, $\Delta A / A_{0}=P / L$ (for $\Omega=Q=4$ ) becomes smaller than $P / L^{*} \approx 0.1$, as $a$ approaches $2 a_{0}$. (c) The graph here shows $\Delta A / A_{0}$ as a function of $Q$, which is chosen to coincide with $\Omega$, for the two choices of $a: a=a_{0}, 2 a_{0}$. It suggests that AMPs become more potent with increasing $Q=\Omega$ and $|H|$ (the magnitude of the hydrophobic free energy). For $\Omega>4, \Omega N_{p} / N_{0}>P / L$. The quantity $\Omega N_{p} / N_{0}$ is a better measure of LPS perturbation, since it is related to the area expansion of the LPS layer induced by peptide binding. The corresponding threshold value is represented by a horizontal dashed line. For $Q \geqslant 6$, the curve with squares in magenta obtained for a dilated lattice, is above the threshold value (the dashed line); also included is a curve obtained for the original lattice, which is well above the dashed line. This may offer design principles for combating resistant bacteria with reduced LPS charges.

$K_{\mathrm{A}}=240 \mathrm{pN} / \mathrm{nm}$ for a bilayer (or $K_{\mathrm{A}}=120 \mathrm{pN} / \mathrm{nm}$ for an LPS monolayer); note that $1 \mathrm{pN} / \mathrm{nm}=1 \mathrm{mN} / \mathrm{m}$. Our choice of $K_{\mathrm{A}}$ is comparable with a recent experimental estimate of $\approx 100 \mathrm{pN} / \mathrm{nm}$ for a LPS monolayer [16] and is similar to that for phospholipids [40,41].

Figure 3 displays our results for the fractional area change $\Delta A / A_{0}=\Omega N_{p} / N_{0}$ [Fig. 3(a)] and lateral pressure [Fig. 3(b)], as a function of [AMP] (left) or $\left[\mathrm{Mg}^{2+}\right]$ (right); also included are the results for $2 N_{2} / N_{0}$ on the right axis in Fig. 3(a). Here [...] is the molar concentration of .... For $\Omega=Q=4$ (as for magainin 2), $\Delta A / A_{0}=Q N_{p} / N_{0}=P / L$.
(Left) First, the graph on the left in Fig. 3(a) shows how AMPs compete with $\mathrm{Mg}^{2+}$ for the LPS layer and perturb the layer for a few choices of $\left[\mathrm{Mg}^{2+}\right]$, the bulk concentration of $\mathrm{Mg}^{2+}:\left[\mathrm{Mg}^{2+}\right]=1,5,10 \mathrm{mM}$. Recall that $P / L^{*} \approx 0.1$ (for magainin 2), i.e., the threshold value of $P / L$ beyond which the LPS layer becomes unstable to rupture [24], marked by the horizontal dashed line. As [AMP] increases, their apparent binding affinity is enhanced, i.e., the occupancy of AMPs on the LPS layer increases (so does $\Delta A / A_{0}$ ) and eventually reaches $P / L^{*}$, more so for smaller $\left[\mathrm{Mg}^{2+}\right]$. This results in a reduced occupancy of $\mathrm{Mg}^{2+}$ on LPS (right axis). The graph 
on the left in Fig. 3(a) suggests that LPS perturbation is more effective for a smaller value of $\left[\mathrm{Mg}^{2+}\right]$.

The results in Fig. 3(a) are consistent with and offer a quantitative basis for the longstanding view that AMPs displace competitively previously-bound $\mathrm{Mg}^{2+}$ ions from the LPS layer, diminishing OM integrity [3-5,10] (see Refs. [19,20] for recent attempts without hydrophobic interactions taken into account).

(Right) The graph on the right in Fig. 3(a) shows the favorable effects of $\left[\mathrm{Mg}^{2+}\right]$ on LPS for a few choices of [AMP], the bulk concentration of AMPs, as indicated in the legend. As $\left[\mathrm{Mg}^{2+}\right]$ increases, their apparent binding affinity also increases. As a result, the amount of $\mathrm{Mg}^{2+}$ on the LPS layer increases, while that of AMPs decreases. The integrity of the LPS layer will be well preserved at a sufficiently high but still physiologically relevant $\left[\mathrm{Mg}^{2+}\right]$, i.e., $\lesssim 10 \mathrm{mM}$, except for the case $[\mathrm{AMP}]=5 \mu \mathrm{M}$, represented by the curve in tangerine.

The graph on the left in Fig. 3(b) summarizes our results for the (excess) lateral pressure $\Delta \Pi$ of the LPS layer induced by peptide binding. This quantity reflects the degree of LPS perturbation [see Eq. (13)] and is measurable experimentally [31]. Our results, obtained for an LPS bilayer, are given in units of $k_{\mathrm{B}} T / \mathrm{nm}^{2}\left(1 k_{\mathrm{B}} T / \mathrm{nm}^{2} \approx 4.1 \mathrm{mN} / \mathrm{m}\right.$ at $\left.300 \mathrm{~K}\right)$.

(Left) The increase of $\Delta \Pi$ with [AMP] is correlated with the $[\mathrm{AMP}]$ dependence of the fractional area increase $\Delta A / A_{0}$ in Fig. 3(a). As [AMP] increases, more peptides will be hydrophobically associated with LPS, increasing $\Delta \Pi$, according to Eq. (13). Consistent with the results in the left figure in Fig. 3(a), the results in this graph suggest that LPS perturbation is more effective for a smaller value of $\left[\mathrm{Mg}^{2+}\right]$.

(Right) This shows how $\Delta \Pi$ varies with $\left[\mathrm{Mg}^{2+}\right]$ for a few choices of [AMP] (see the legend). Obviously, it decreases as $\left[\mathrm{Mg}^{2+}\right]$ increases. This is paralleled by the enhanced binding of $\mathrm{Mg}^{2+}$ ions and reduced binding of AMPs at higher $\mathrm{Mg}^{2+}$ concentrations seen in Fig. 3(a).

The general trend shown in Fig. 3(b) is consistent with what was observed for different peptides (e.g., polymyxin B and polymyxin E1 for which $Q=6$ ) [31]: a peptide-induced lateral pressure, which was reduced in the presence of $\mathrm{Mg}^{2+}$. This comparison is not so conclusive because of the biological uncertainties about these peptides and their interaction with LPS. Recall that this work is focused on magainin 2, since its parameters are relatively well characterized for the biophysical modeling of its interaction with LPS [19,20,24]. Nevertheless, what is clear is that the results in Fig. 3 capture the essential features of competing effects on LPS of cationic amphiphilic peptides and $\mathrm{Mg}^{2+}$.

The results in Fig. 3 imply that under the right conditions (i.e., low $\left[\mathrm{Mg}^{2+}\right]$ and high [AMP]) cationic AMPs such as magainin 2 can readily disrupt the outer, LPS layer of the $\mathrm{OM}$, eventually leading to OM permeabilization. Bacterial sensitivity to a given peptide also depends on peptide-LPS parameters. A systematic consideration of how peptide-LPS parameters are implicated in peptide activity will be useful for developing optimized peptides as new peptide antibiotics [50,51]. Conversely, it will offer biophysical insights into bacterial strategies for developing resistance against AMPs $[10,11,33,35]$; indeed, (partial) neutralization of LPS charges weakens AMP's attraction to the cell surface of Gram-negative bacteria, lowering $P / L$.
It is worth noting that $Q$ can be varied while other peptide parameter are held fixed [27,52-54]. This justifies our consideration of peptide-parameter-activity relationships. Some subtlety, however, arises regarding the ion-pairing energy: $\ell_{B} \Delta_{\varepsilon} Q / \delta_{p}$. In our original approach [see Fig. 1, Fig. 2 , and Eq. (8)], each peptide charge is assumed to occupy one lattice site. The length of a peptide or $\Omega$ sets the maximum number of ion pairs that the peptide forms with LPS charges. For $Q<\Omega, Q$ can be varied independently of $\Omega$. For $Q>\Omega$, not all peptide charges can pair with LPS charges. In this case, it suffices to use $\Omega$ in place of $Q$ in the ion-pairing energy. Similarly, the ion-pairing energy can be modified as the lattice dilates: if $a_{0} \rightarrow a=\alpha a_{0}(\alpha=1,2,3, .$.$) , the number of ion$ pairs diminishes by the same factor $\alpha$. This is equivalent to changing $\delta_{p}$ to $\alpha \delta_{p}$.

Figure 4 displays our results for the fractional area change $\triangle A / A_{0}$ and the occupancy of $\mathrm{Mg}^{2+}$ versus $Q$ (A and C) and $a / a_{0}$ (B). We have chosen $\Omega=4$ in both (A) and (B); $Q=1, \ldots, 7$ in (A) and $Q=4$ in (B); in (B), we have changed $a_{0}$ to $\alpha a_{0}=\{1,2,3,4\} a_{0} ;\left[\mathrm{Na}^{+}\right]=100 \mathrm{mM}$ in all cases (other parameters are the same as in Fig. 3). Note that $\Delta A / A_{0}=$ $\Omega N_{p} / N_{0}(=P / L$ for $\Omega=Q=4)$.

As indicated by Fig. 4(a), as $Q$ increases, $\Delta A / A_{0}$ increases initially and then decreases beyond $Q=4$, for all $\left[\mathrm{Mg}^{2+}\right]$ values used: $\left[\mathrm{Mg}^{2+}\right]=1,5,10 \mathrm{mM}$. In contrast, the fractional charge occupancy of $\mathrm{Mg}^{2+}$, i.e., $2 N_{2} / N_{0}$, decreases monotonically with $Q$. The nonmonotonic dependence of $\triangle A / A_{0}$ on $Q$ can be attributed to the balance between the two competing effects: LPS-peptide attractions and peptidepeptide repulsions on the LPS surface, both stronger for a larger $Q$ value. This appears to be a common feature of electrostatic binding. Similarly to what was seen with phospholipid membranes [27,28], there exists an optimal charge at which peptide active against LPS is maximized.

As the lattice dilates [Fig. 4(b)], both $\Delta A / A_{0}$ and $2 N_{2} / N_{0}$ decrease monotonically, as their attraction with LPS diminishes. In all the cases shown, $\Delta A / A_{0}=Q N_{p} / N_{0}=P / L$ (for $\Omega=Q=4$ ) becomes smaller than $P / L^{*}$, as $a=\alpha a_{0}$ approaches $2 a_{0}$, i.e., if every other LPS charges are neutralized. This shows to what extent Gram-negative bacteria should alter their LPS charges to induce resistance to cationic peptides.

What is the design principle for combating bacteria with reduced LPS charges? To answer this, we have varied the peptide parameters as follows: $Q=\Omega$ and $|H|$ in proportion with each other so that $H=-10 k_{\mathrm{B}} T$ for $Q=4$; it suffices to choose $H=2.5 Q k_{\mathrm{B}} T$. This is based on the expectation that the magnitude of the hydrophobic free energy $|H|$ is proportional to peptide length $\Omega a$. Also a natural choice of $g$ in this consideration is $g=Q=\Omega$.

The graph in Fig. 4(c) shows the results for $\Delta A / A_{0}$ obtained this way for the two choices of $a: a=a_{0}, 2 a_{0}$. It suggests that the peptide becomes more potent with increasing $Q=\Omega$ and $|H|$, simultaneously. Note that for $\Omega>4, \Omega N_{p} / N_{0}>P / L$. The quantity $\Omega N_{p} / N_{0}$ coincides with $\Delta A / A_{0}$ and is a better measure of LPS perturbation than $P / L$. The threshold value $\left(\Omega N_{p} / N_{0}=0.1\right)$ is represented by a horizontal dashed line. For $Q \geqslant 6$, the curve with squares in magenta, obtained for a dilated lattice, is above the threshold value required for LPS permeabilization (the dashed line); so is the curve obtained for the original lattice. 
Further considerations along the line of our attempts in Fig. 4 will be useful for offering design principles for optimizing peptide sequences against resistant bacteria, especially with reduced LPS charges. First, note that the fractional area expansion $\triangle A / A_{0}$ is not the sole determinant of LPS disruption. The free energy of pore-forming peptides depends on peptide size or length, among others. For instance, a possible hydrophobic mismatch between peptides and lipid tails will influence the energetics of pore formation. (The fractional area change $\triangle A / A_{0}$ still remains relevant and measures the degree of LPS perturbation, prior to pore formation).

Also, peptide potency against different membranes will not be necessarily correlated with each other. While LPS is unique to the bacterial outer membrane, phospholipids are a major component of the cytoplasmic membranes of both bacterial and eukaryotic cells (see Ref. [55] and references therein); also cholesterol present in the latter modifies their biophysical properties and interactions with amphiphilic molecules [10,41]; if the bacterial cytoplasmic membrane is rich in anionic lipids (e.g., phosphatidylglycerol), the eukaryotic counterpart is neutral on the outer layer. Importantly, it has recently be shown that the cell selectivity of AMPs depends not only on peptide-membrane parameters but also on cell concentrations [56,57]. How all these biological details contribute toward peptide selectivity is unclear but can be further clarified.

\section{CONCLUSIONS}

In conclusion, we have presented a coarse-grained model for LPS perturbation by antimicrobial peptides (AMPs), in an electrolyte solution containing $\mathrm{Mg}^{2+}$ ions. To treat simultaneously the hydrophobic and electrostatic interactions of LPS with AMPs, we have introduced a reconstructed-lattice model of LPS layers [see Fig. 1(c)]. The main results based on our approach are summarized below.

In parameter ranges of biological relevance, the antimicrobial peptide magainin 2 effectively disrupts LPS layers by hydrophobically inserting into the layer, initially in a parallel orientation (Fig. 3). It competes with $\mathrm{Mg}^{2+}$ for LPS and diminishes $\mathrm{Mg}^{2+}$-binding of LPS. At a few micro-molar concentrations, the peptide coverage reaches a threshold value $P / L^{*}$ required for LPS rupture. At low concentrations of the peptide $(\lesssim 0.1 \mu \mathrm{M})$; however, the competition becomes favorable to $\widetilde{\mathrm{Mg}}^{2+}$, resulting in peptide binding below $P / L^{*}$ $(\approx 0.1)$, as long as $\mathrm{Mg}^{2+} \gtrsim 2 \mathrm{mM}$.

Similar to what was observed for phospholipid membranes [27,28], there exists an optimal peptide charge for perturbing LPS layers [Fig. 4(a)]. This is a general feature of electrostatic interactions between cationic peptides and anionic lipids, even though the precise value of the optimal charge can be modeldependent. Increasing $Q$ from $Q \approx 1$ will initially enhance peptide binding but eventually diminish it, as the repulsion between bound peptides catches up with their attraction with lipid charges.

Also in typical parameter ranges, LPS layers with charges reduced by half can remain intact [Fig. 4(b)], in contrast to the corresponding fully charged LPS layer, which is well above $P / L^{*}$ for magainin $2(Q=4)$. Finally, adjusting the peptide parameters $\Omega, Q$, and $H$ in proportion with each other appears to be a good strategy for optimizing peptide activity against the LPS layers with reduced charges [Fig. 4(c)]. However, this is solely based on the area expansion $\Delta A / A_{0}$ induced by peptide binding. For a more complete picture, however, the energetics of pore formation will have to be taken into account. A hydrophobic mismatch between peptides and lipids, which is peptide-length dependent, will change peptide's propensity to form a pore and may have an unexpected consequence.

Thanks to their outer membrane, Gram-negative bacteria have become an increasingly serious source for infection [17]. AMPs must cross the OM to reach other targets such as the inner, cytoplasmic membrane and intracellular biomolecules. In the case of the human antimicrobial LL-37, the significance of crossing the OM has recently been revealed: it is temporally correlated with peptide's bacteriostatic activity [58]. Use of a more realistic model of LPS will be beneficial for clarifying further OM permeability. Indeed, recent experiments suggest that uncharged sugar groups in LPS reduce the electrostatic binding of cationic antimicrobials to LPS [14]. In principle, this effect can be captured in our approach [Eq. (8)] by considering the LPS molecules as forming a polymer brush.

Nanoparticle inclusion in a polymer brush has recently been studied numerically and theoretically $[59,60]$. In particular, the inclusion free energy or the excess free energy due to single inclusion $\Delta F_{\text {brush }}$ was obtained for a spherical particle. If $R$ is the radius of the inclusion and $\sigma$ the grafting density, $\Delta F_{\text {brush }}$ is given by $\Delta F_{\text {brush }} \approx A \sigma^{3 / 2} R^{3}+B R^{2}$, where $A$ and $B$ are constants [59]. The applicability of this result to an LPS-peptide system is, however, unclear, because of the conformational degrees of freedom stored in a polymer coil, but not in a simple sphere. For $\alpha$-helical or cylindrical peptides, it is tempting to use the peptide volume $v_{p}$ in place of $R^{3}: \Delta F_{\text {brush }} \approx A \sigma^{3 / 2} v_{p}+B v_{p}^{2 / 3}$. The numerical prefactor of $\Delta F_{\text {brush }}$, however, depends on molecular details (e.g., peptide structure); the values of $A$ and $B$ can be different from those for spherical inclusions and should reflect the interactions of peptides with the surrounding polysaccharides.

If peptides do not aggregate, for small $N_{p} / N_{0}$, the excess free energy of $N_{p}$ peptides in a brush is simply $N_{p} \times \Delta F_{\text {brush }}$, but for large $N_{p} / N_{0}$, it will have a nonlinear dependence on $N_{p}$.

If peptides in the brush are modeled as (self-avoiding) random coils, a polymer-physics approach will be applicable. A standard approach to calculating the free energy of a "semidilute" polymer solution is to count the number of "blobs" and assign $k_{\mathrm{B}} T$ to each [61]; inside each blob, chain statistics remains unchanged by the presence of neighboring chains. In a homogeneous polymer solution, the blob size $\xi$ is related to the volume fraction of monomers $\phi$ as $\xi \approx \phi^{-3 / 4}$. In a binary mixture, $\phi$ should be interpreted as the total volume fraction of monomers. Let $\phi_{0}$ be the volume fraction of monomers in the absence of peptides (i.e., that of LPS monomers) and $\phi_{p}$ the volume fraction of peptide monomers. Assuming that the combined system is in a semidilute regime, the excess free energy per peptide can be obtained as $\Delta F_{\text {brush }} \approx$ $\left(\phi_{0}+\phi_{p}\right)^{9 / 4}-\phi_{0}^{9 / 4}$, where numerical prefactors are omitted.

In reality, the conformational properties of peptides in the LPS brush will likely combine both features of rigid cylinders and random coils. While a fuller picture is lacking, this line 
of reasoning is consistent with the finding that the presence of uncharged sugar groups in the outer core of LPS weakens the binding of cationic antimicrobials to the LPS [14]. A more quantitative study of peptide inclusion in an LPS brush will be useful for improving a coarse-grained approach.

\section{ACKNOWLEDGMENTS}

This work was supported by the Natural Sciences and Engineering Research Council (NSERC) of Canada. We acknowledge useful discussions with R. Hancock.

\section{APPENDIX}

In this Appendix, we examine a hypothetical LPS bilayer, for which the deformation free energy caused by peptide binding is identical to what we would expect from a phospholipid bilayer. Indeed, the compression modulus is similar for both LPS and phospholipid bilayers, as discussed in the main text; in both cases, the deformation free energy is expected to be linear with $N_{p}$ for small $N_{p}$, but as $N_{p}$ increases it will crossover to a quadratic (harmonic) form. Importantly, in both cases, a threshold peptide coverage is required for peptide insertion in a perpendicular orientation; beyond this, a certain fraction of bound peptides form pores in the bilayer. This is evidence that the deformation free energy is quadratic with $N_{p}$ in the parameter range of biological significance. What remains unclear is when the crossover occurs. As evidenced below, largely independent of this, for practical purposes, the harmonic deformation free energy may be used.

We have followed a theoretical approach based on a onedimensional arrangement of bound peptides [39]. The resulting (total) deformation free energy (in units of $k_{\mathrm{B}} T$ ), which shows a crossover from small- $N_{p}$ to large- $N_{p}$ limits [39], can be expressed as

$$
F\left(N_{p}\right)=\frac{1}{2} a^{2} K_{\mathrm{A}} Q^{2} \frac{N_{p}}{2 \sqrt{2} \lambda} \frac{\sinh \left(\frac{1}{\sqrt{2} \lambda x_{p}}\right)+\sin \left(\frac{1}{\sqrt{2} \lambda x_{p}}\right)}{\cosh \left(\frac{1}{\sqrt{2} \lambda x_{p}}\right)-\cos \left(\frac{1}{\sqrt{2} \lambda x_{p}}\right)} .
$$

Here $x_{p}=N_{p} / N_{0}$ is the fractional occupancy of peptides and the parameter $\lambda$ is given by $\lambda=13 \AA$ for a bilayer [39].

Note that the free energy in Eq. (A1) becomes linear with $N_{p}$ as $N_{p} \rightarrow 0: F \approx \frac{1}{2} a^{2} K_{\mathrm{A}} Q^{2} \frac{N_{p}}{2 \sqrt{2} \lambda}$. As $N_{p}$ increases, it crosses over to the harmonic deformation term in Eq. 8: $F \approx \frac{1}{2} a^{2} K_{\mathrm{A}} Q^{2} N_{p}^{2} / N_{0}$, which is identical to the expression in Eq. (7). It shows the expected crossover between the linear and quadratic free energy.

We have solved Eq. (8) for $N_{p}$ using the two choices of the deformation free energy: the original harmonic free energy given in Eq. (8) and the full expression in Eq. (A1) in place of the harmonic one. Figure 5 shows our results for the fractional

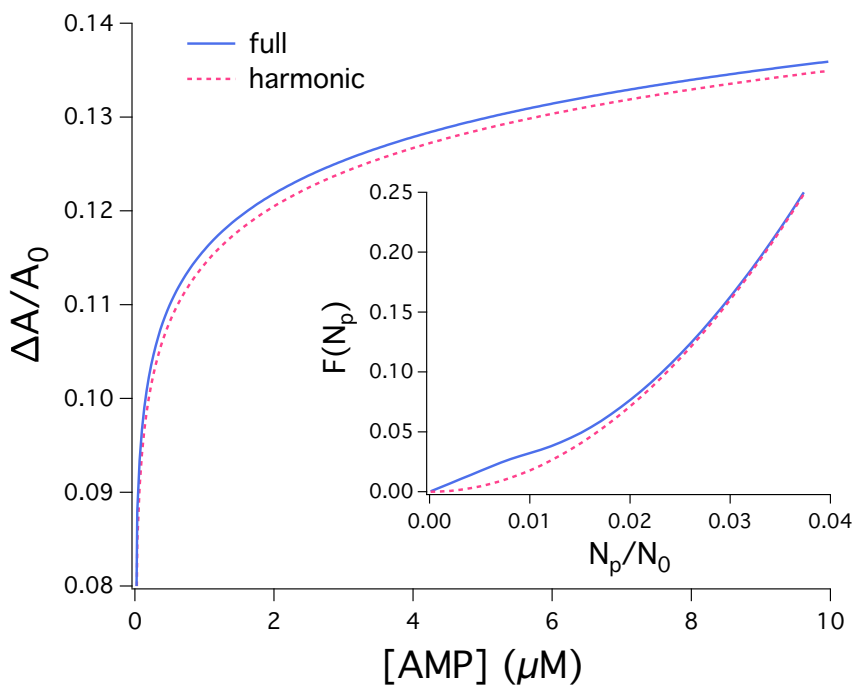

FIG. 5. Fractional area change $\Delta A / A_{0}$ obtained using the two choices of the deformation free energy: the original harmonic free energy given in Eq. (8) and the full expression in Eq. (A1) in place of the harmonic one, described by the curves in magenta (dashed) and blue (solid), respectively. We have chosen the parameters as $\left[\mathrm{Na}^{+}\right]=$ $100 \mathrm{mM}$ and $\left[\mathrm{Mg}^{2+}\right]=5 \mathrm{mM}$; other parameters are the same as in Fig. 3. The difference between the two cases seems to be minor for the entire range of [AMP]. Shown in the inset is the free energy in Eq. (A1) (in blue) and its harmonic approximation (in magenta).

area change $\Delta A / A_{0}=Q N_{p} / N_{0}$; the blue (solid) curve represents the full free energy and the magenta (dashed) the harmonic approximation. We have chosen $\left[\mathrm{Na}^{+}\right]=100 \mathrm{mM}$ $\left(\kappa^{-1} \approx 10 \AA\right)$ and $\left[\mathrm{Mg}^{2+}\right]=5 \mathrm{mM}$; other parameters are the same as in Fig. 3. The difference between the two cases (blue and magenta) seems to be insignificant for the entire range of [AMP] (e.g., about $2 \%$ when $\Delta A / A_{0} \approx 0.1$ ).

Also included is a graph of $F\left(N_{p}\right)$ (see the inset). The difference in $\Delta A / A_{0}=Q N_{p} / N_{0}$ can be attributed to the difference in $F\left(N_{p}\right)$ between the two cases: the harmonic free energy and the crossover free energy. For $x_{p}=N_{p} / N_{0} \gtrsim$ 0.08 , the harmonic free energy suppresses peptide binding more effectively: for this range of $x_{p}, \partial F\left(N_{p}\right) / \partial N_{p}$ is slightly larger for the harmonic approximation.

Our analysis presented here suggests that for practical purposes the harmonic deformation free energy can be used for the parameter ranges of biological interest, e.g., for the entire range shown in Fig. 5. The general agreement between the two cases means that this conclusion is largely independent of the details of Eq. (A1). It justifies the use of this free energy in Eq. (8). For further considerations, a better understanding of $\lambda$ will be needed.
[1] C. R. H. Raetz and C. Whitfield, Lipopolysaccharide endotoxins, Annu. Rev. Biochem. 71, 635 (2002).

[2] S. G. Wilkinson, Bacterial lipopolysaccharides-Themes and variations, Prog. Lipid Res. 35, 283 (1996).

[3] H. Nikaido, Molecular basis of bacterial outer membrane permeability revisited, Microbiol. Mol. Biol. Rev. 67, 593 (2003).
[4] R. E. W. Hancock, Alterations in outer membrane permeability, Ann. Rev. Microbiol. 38, 237 (1984).

[5] R. E. W. Hancock, The bacterial outer membrane as a drug barrier, Trends Microbiol. 5, 37 (1997).

[6] M. Vaara, Agents that increase the permeability of the outer membrane, Microbiol. Rev. 56, 395 (1992). 
[7] K. Matsuzaki, K. Sugishita, and K. Miyajima, Interactions of an antimicrobial peptide, magainin 2, with lipopolysaccharidecontaining liposomes as a model for outer membranes of Gramnegative bacteria, FEBS Lett. 449, 221 (1999).

[8] L. Leive, Release of lipopolysaccharide by EDTA treatment of E. coli, Biochem. Biophys. Res. Commun. 21, 290 (1965).

[9] A.H. Delcour, Outer membrane permeability and antibiotic resistance, Biochim. Biophys. Acta 1794, 808 (2009).

[10] M. Zasloff, Antimicrobial peptides of multicellular organisms, Nature 415, 389 (2002).

[11] K. A. Brogden, Antimicrobial peptides: Pore formers or metabolic inhibitor, Nat. Rev. Microbiol. 3, 238 (2005).

[12] L. A. Clifton et al., An accurate in vitro model of the $E$. coli envelope, Angew. Chem. 127, 12120 (2015).

[13] L. A. Clifton et al., Effect of divalent cation removal on the structure of gram-negative bacterial outer membrane models, Langmuir 31, 404 (2015).

[14] L. A. Clifton et al., The effect of lipopolysaccharide core oligosaccharide size on the electrostatic binding of antimicrobial proteins to models of the gram negative bacterial outer membrane, Langmuir 32, 3485 (2016).

[15] Jr. A. Nascimento, F. J. Pontes, R. Lins, and T. A. Soares, Hydration, ionic valence and cross-linking propensities of cations determine the stability of lipopolysaccharide (LPS) membranes, Chem. Commun. 50, 231 (2014).

[16] M. Herrmann, E. Schneck, T. Gutsmann, K. Brandenburg, and M. Tanaka, Bacterial lipopolysaccharides form physically crosslinked, two-dimensional gels in the presence of divalent cations, Soft Matter 11, 6037 (2015).

[17] D. M. Livermore, Fourteen years in resistance, Int. J. Antimicrob. Agents 39, 283 (2012).

[18] K. A. Dill and S. Bromberg, Molecular Driving Forces: Statistical Thermodynamics in Biology, Chemistry, Physics, and Nanoscience, 2nd ed. (Garland Science, New York, 2010).

[19] N. Lam, Z. Ma, and B.-Y. Ha, Electrostatic modification of the lipopolysaccharide layer: Competing effects of divalent cations and polycationic or polyanionic molecules, Soft Matter 10, 7528 (2014).

[20] N. H. Lam and B.-Y. Ha, Surface-lattice model describes electrostatic interactions of ions and polycations with bacterial lipopolysaccharides: Ion valence and polycation's excluded area, Langmuir 30, 13631 (2014).

[21] S. Taheri-Araghi and B.-Y. Ha, Electrostatic bending of lipid membranes: How are lipid and electrostatic properties interrelated? Langmuir 26, 14737 (2010).

[22] M. L. Henle, C. D. Santangelo, D. M. Patel, and P. A. Pincus, Distribution of counterions near discretely charged planes and rods, Europhys. Lett. 66, 284 (2004).

[23] A. Travesset and D. Vaknin, Bjerrum pairing correlations at charged interfaces, Europhys. Lett. 74, 181 (2006).

[24] L. Ding, L. Yang, T. M. Weiss, A. J. Waring, R. I. Lehrer, and H. W. Huang, Interaction of antimicrobial peptides with lipopolysaccharides, Biochemistry 42, 12251 (2003).

[25] M.-T. Lee, F.-Y. Chen, and H. W. Huang, Energetics of pore formation induced by membrane active peptides, Biochemistry 43, 3590 (2004).

[26] H. W. Huang and N. E. Charron, Understanding membraneactive antimicrobial peptides, Q. Rev. Biophys. 50, e10 (2017).

[27] M. Dathe, H. Nikolenko, J. Meyer, M. Beyermann, and M. Bienert, Optimization of the antimicrobial activity of magainin peptides by modification of charge, FEBS Lett. 501, 146 (2001).

[28] S. Taheri-Araghi and B.-Y. Ha, Cationic antimicrobial peptides: A physical basis for their selective membrane-disrupting activity, Soft Matter 6, 1933 (2010).

[29] Computer simulations have been used to study LPS systems $[15,62]$. However, simulating an LPS-peptide-ion system, possibly with various parameter choices, is computationally demanding. An LPS-ion system simulated recently consists of less than 100 LPS molecules [15]. The computational power needed for the current problem is prohibitively demanding.

[30] E. Schneck, T. Schubert, O. V. Konovalov, B. E. Quinn, T. Gutsmann, K. Brandenburg, R. G. Oliveira, D. A. Pink, and M. Tanaka, Quantitative determination of ion distributions in bacterial lipopolysaccharide membranes by grazing-incidence X-ray fluorescence, Proc. Natl. Acad. Sci. USA 107, 9147 (2010).

[31] L. Zhang, P. Dhillon, H. Yan, S. Farmer, and R. E. W. Hancock, Interactions of bacterial cationic peptide antibiotics with outer and cytoplasmic membranes of pseudomonas aeruginosa, Antimicrob. Agents Chemother. 44, 3317 (2000).

[32] R. C. Chatelier and A. P. Minton, Adsorption of globular proteins on locally planar surfaces: Models for the effect of excluded surface area and aggregation of adsorbed protein on adsorption equilibria, Biophys. J. 71, 2367 (1996).

[33] L. Guo, K. B. Lim, C. M. Poduje, M. Daniel, J. S. Gunn, M. Hackett, and S. I. Miller, Lipid A Acylation and Bacterial Resistance against Vertebrate Antimicrobial Peptides, Cell 95, 189 (1998).

[34] E. Schneck, R. G. Oliveira, F. Rehfeldt, B. Demé, K. Brandenburg, U. Seydel, and M. Tanaka, Mechanical properties of interacting lipopolysaccharide membranes from bacteria mutants studied by specular and off-specular neutron scattering, Phys. Rev. E 80, 041929 (2009).

[35] A. Peschel and H.-G. Sahl, The co-evolution of host cationic antimicrobial peptides and microbial resistance, Nat. Rev. Microbiol. 4, 529 (2006).

[36] For LPS Re, this approach works well if $\Omega=4$. Each peptide can then be considered as shifting one LPS molecule. For $\Omega \neq Q$, $\Omega$ can be viewed as a tuning parameter, which is to be adjusted for the best fit to available data. Because of the non-specific nature of intermolecular interactions involved (electrostatic and hydrophobic), we believe that our lattice model is more reliable than it may seem at first glance, largely independent of its details. See Sec. III for relevant discussions.

[37] S. Taheri-Araghi and B.-Y. Ha, Charge renormalization and inversion of a highly charged lipid bilayer: Effects of dielectric discontinuities and charge correlations, Phys. Rev. E. 72, 021508 (2005).

[38] This can be most readily seen at the mean-field level [19,37]. For $\varepsilon_{w} / \varepsilon_{l} \gg 1$, however, one can show that this is more generally correct, as long as all surface charges, including lipid charges and those on bound ions, are treated on equal footing [37]. One possible way to see this is to take a large- $\varepsilon_{w} / \varepsilon_{l}$ limit in Eq. (15) in Ref. [37]. The effect of dielectric discontinuity can be factored out as in Eq. (1) in our work.

[39] H. W. Huang, Elasticity of lipid bilayer interacting with amphiphilic helical peptides, Phys. II France 5, 1427 (1995). 
[40] W. Rawicz, K. C. Olbrich, T. McIntosh, D. Needham, and E. Evans, Effect of chain length and unsaturation on elasticity of lipid bilayers, Biophys. J. 79, 328 (2000).

[41] D. Boal, Mechanics of the Cell, 2nd ed. (Cambridge University Press, Cambridge, 2011).

[42] T. L. Hill, An Introduction to Statistical Thermodynamics (Dover Publications, London, 1986).

[43] Let $A_{p}$ be the area each peptide adds to the LPS layer. If we assume that the LPS and inner layers have the same elastic properties and they remain flat, for an asymmetric binding, the bilayer area will increase by $N_{p} A_{p} / 2$. One can then establish the following equality: $N_{0} a^{2}+N_{p} A_{p}=N_{0} a_{0}^{2}+N_{p} A_{p} / 2$. If we use $A_{p}=\Omega a$, we arrive at the relation in Eq. (12). On the other hand, if we insist on using $A_{p}=\Omega a_{0}^{2}$, we obtain $a=$ $a_{0} \sqrt{1-\Omega N_{p} / 2 N_{0}}$. For a symmetric distribution of AMPs in an LPS bilayer that we focus on, $a$ can be approximated by $a_{0}$.

[44] Equation (13) is complete for an LPS monolayer or a symmetric LPS bilayer. For an asymmetrical bilayer (e.g., the OM), the inner layer makes a distinct contribution. Nevertheless, it is still useful to focus on the LPS layer, as described by Eq. (13), since it will essentially determine the permeability of the LPS layer, which can be considered separately from the inner layer [24].

[45] J. N. Israelachvili, Intermolecular and Surface Forces, 3rd ed. (Academic Press, San Diego, 2011).

[46] Note that $r_{i}(i=1,2)$ here is the "hydrated" radius [45], which is larger than the corresponding "bare" ion radius; ions are swollen by hydration. This is also different from $\delta_{i}$, i.e., ion size on the LPS surface, since ion binding will diminish the degree of hydration. The value of $\delta_{i}$ chosen here falls between the bare and hydrated values.

[47] This value, employed recently [19,20], can be obtained if the peptide is viewed as a cylinder of radius $0.6 \mathrm{~nm}$ and length $2.2 \mathrm{~nm}$. In reality, it depends on peptide sequences and the number of amino acids; in the bulk, the peptide is better represented as a random coil. But the choice of $v_{p}$ is not so crucial. Here we use a typical value, consistent with Ref. [49].

[48] For the case of phospholipids, the area of each peptide on the LPS layer, more precisely, an area each peptide adds to the layer, $A_{p}=\Omega a^{2}$, depends not only on peptide-membrane parameters but also on the hydration of lipid headgroups [25]; as a result, there is no simple way to determine $A_{p}$. Our choice $\Omega=4$ (thus $A_{p} \approx 164 \AA^{2}$ ) is similar to that for magainin 2 on a typical bacterial phospholipid membrane [25]. It is, however, the combination of $K_{\mathrm{A}} \times A_{p}^{2}$ that enters into $F_{\text {deform }}$ in Eq. 7; adjusting $K_{\mathrm{A}}$ to $K_{\mathrm{A}} / 2$ is equivalent to decreasing $A_{p}$ by $1 / \sqrt{2}$. On the other hand, other terms in Eq. (8) are typically not as sensitive to $A_{p}$ as $F_{\text {deform }}$.
[49] A. Zemel, A. Ben-Shaul, and M. May, Perturbation of a lipid membrane by amphipathic peptides and its role in pore formation, Eur. Biophys. J. 34, 230 (2005).

[50] R. E. W. Hancock and H.-G. Sahl, Antimicrobial and hostdefense peptides as new anti-infective therapeutic strategies, Nat. Biotech. 24, 1551 (2006).

[51] C. D. Fjell, J. A. Hiss, R. E. W. Hancock, and G. Schneider, Designing antimicrobial peptides: Form follows function, Nat. Rev. Drug Discov. 11, 37 (2012).

[52] A. Giangaspero, L. Sandri, and A. Tossi, Amphipathic $\alpha$ helical antimicrobial peptides: A systematic study of the effects of structural and physical properties on biological activity, Eur. J. Biochem. 268, 5589 (2001).

[53] I. Zelezetsky and A. Tossi, $\alpha$-helical antimicrobial peptidesUsing a sequence template to guide structure-activity relationship studies, Biochim. Biophys. Acta 1758, 1436 (2006).

[54] Z. Jiang, A. I. Vasil, J. D. Hale, R. E. Hancock, M. L. Vasil, and R. S. Hodges, Effects of net charge and the number of positively charged residues on the biological activity of amphipathic $\alpha$ helical cationic antimicrobial peptides, Biopolymers 90, 369 (2008).

[55] R. M. Epand and R. F. Epand, "Biophysical Analysis of Membrane-Targeting Antimicrobial Peptides: Membrane Properties and the Design of Peptides Specifically Targeting Gramnegative Bacteria," in Antimicrobial Peptides: Discovery, Design and Novel Therapeutic Strategies, edited by G. Wang (CABI, UK, 2010).

[56] A. Bagheri, S. Taheri-Araghi, and B.-Y. Ha, How cell concentrations are implicated in cell selectivity of antimicrobial peptides, Langmuir 31, 8052 (2015).

[57] K. Matsuzaki, Control of cell selectivity of antimicrobial peptides, Biochim. Biophys. Acta, Biomembr. 1788, 1687 (2009).

[58] K. A. Sochacki, K. J. Barns, T. Bucki, and J. C. Weisshaar, Real-time attack on single Escherichia coli cells by the human antimicrobial peptide LL-37, Proc. Natl. Acad. Sci. USA 108, E77 (2011).

[59] H. Merlitz, C.-X. Wu, and J.-U. Sommer, Inclusion free energy of nanoparticles in polymer brushes, Macromolecules 45, 8494 (2012).

[60] A. Milchev, D. I. Dimitrov, and K. Binder, Excess free energy of nanoparticles in a polymer brush, Polymer 49, 3611 (2008).

[61] P.-G. Gennes, Scaling Concepts in Polymer Physics (Cornell University Press, Ithaca, NY, 1979).

[62] D. S. Patel et al., Dynamics and interactions of OmpF and LPS: influence on pore accessibility and ion permeability, Biophys. J. 110, 930 (2016). 